\title{
Tendencias de la jurisprudencia constitucional colombiana sobre exclusiones del plan de beneficios en salud*
}

Tendencies in the Colombian constitutional case-law regarding health benefits plan exclusions

Tendências da jurisprudência constitucional colombiana sobre as eliminações de serviços do plano de benefícios de saúde

Tendances de la jurisprudence constitutionnelle colombienne sur les exclusions du régime d'avantages sociaux de santé

\section{Christian David Rivera Aguirre (iD}

Abogado. Especialista en Gerencia de la Seguridad Social.

Magíster en Seguridad Social, Universidad Ces

Investigador del Grupo de Estudios Jurídicos

Universidad CES, Medellín - Colombia

Correo electrónico: christianda123@hotmail.com

Artículo de reflexión elaborado a partir del análisis de la jurisprudencia de la Corte Constitucional de Colombia 


\section{Resumen}

El presente artículo presenta las tendencias de la jurisprudencia de la Corte Constitucional colombiana frente al listado de servicios excluidos del Plan de Beneficios en Salud establecido en la Ley 1751 de 2015 Estatutaria de Salud.

Se realizó un rastreo en la página web de la Corte Constitucional, en busca delos pronunciamientos sobre cada uno de los servicios excluidos por la ley, y se aplicó una metodología de ingeniería inversa en las sentencias más recientes, para así identificar el precedente judicial y las tendencias en las decisiones.

Se concluyó que, pese a existir un listado taxativo de exclusiones en la Ley Estatutaria de Salud, la Corte Constitucional lo ha flexibilizado al incorporar reglas bajo las cuales considera procedente ordenar su cubrimiento por el sistema de salud.

\section{Palabras clave}

Corte Constitucional, Plan de beneficios en salud, exclusiones, precedente judicial, Ley 1751 de 2015.

\section{Abstract}

The present paper presents the case-law tendencies of the Corte Constitucional concerning the list of services excluded from the health benefits plan, established by the Ley 1751 de 2015 Estatutaria de Salud.

A search was carried out in the Corte Constitucional's web page in order to find the pronouncements on each of the services excluded by the law, while applying a reverse engineering methodology on the most recent court rulings, to then identify the judicial precedent and tendencies of the decisions.

It was concluded that despite the existence of a restrictive list of exclusions in the Statutory Health Law, the Corte Constitucional has made it flexible by incorporating rules under which it is considered appropriate to order coverage by the health system.

Cómo citar este artículo:

Rivera, C. (2019). Tendencias de la jurisprudencia constitucional colombiana sobre exclusiones del plan de beneficios en salud. Revista de la Facultad de Derecho y Ciencias Políticas, 49(130), pp. 102-124. doi: http://dx.doi.org/10.18566/rfdcp.v49n130.a05

Recibido: 30 de octubre de 2018.

Aprobado: 17 de mayo de 2019. 


\section{Key Words}

Constitutional Court; Health benefits plan; Exclusions; Judicial precedent; Ley 1751 de 2015.

\section{Resumo}

Esse artigo apresenta as tendências da jurisprudência da Corte Constitucional Colombiana diante da lista de serviços que foram eliminados do Plano de Benefícios de Saúde a partir da Lei estatutária de saúde 1751 de 2015.

Realizou-se uma pesquisa na página web da Corte Constitucional, em busca dos pronunciamentos sobre cada um dos serviços eliminados pela lei; deste modo, aplicou-se uma metodologia de engenharia inversa nas sentenças mais novas, a fim de identificar o precedente judicial e as tendências nas decisões.

Conclui-se que, embora exista uma lista taxativa de eliminações de serviços na Lei Estatutária de Saúde, a Corte Constitucional a tem flexibilizado, logo depois de introduzir as regras sob as quais considera procedente mandar a proteção dos serviços pelo sistema de saúde.

\section{Palabras-chaves}

Corte Constitucional, Plano de Benefícios de Saúde, eliminações de serviços, precedente judicial, Lei 1751 de 2015.

\section{Résumé}

Cet article présente les tendances de la jurisprudence de la Cour constitutionnelle à propos de la liste des services exclus du régime d’avantages sociaux de santé établis par la Loi 1751 de 2015 Statutaire de Santé.

Pour cela, on a fait un suivi de la page web de la Cour constitutionnelle, à la recherche des décisions à propos de chaque service exclu par la loi, en appliquant une méthodologie d'ingénierie inverse sur les sentences les plus récentes pour ainsi identifier le précédent juridique et les tendances des décisions.

Cet article conclut que, malgré l'existence d'une liste restrictive d'exclusions dans la Loi Statutaire de santé, la Cour Constitutionnelle l'a flexibilisé en incorporant des règles sous lesquelles elle considère appropriée sa couverture par le système de santé. 


\section{Mots clés}

Cour constitutionnelle, Régime d'avantages sociaux de santé, exclusions, précédent juridique, Loi 1751 de 2015

\section{Introducción}

Con la promulgación de la Ley 100 de 1993 se establecieron, en Colombia, una serie de coberturas asumidas por el Sistema General de Seguridad Social en Salud (SGSSS), e igualmente se fijaron exclusiones del mismo y, entre el plan y las exclusiones, quedaron las prestaciones no cubiertas y no expresamente excluidas, frente a las cuales se desarrolló un procedimiento para determinar, en cada caso puntual, su aprobación o negación.

Desde la Resolución 5261 de 1994 se instauró un listado de 15 prestaciones expresamente excluidas del sistema, por no contribuir al tratamiento de la enfermedad, entrelas cuales se encontraban los tratamientos confines estéticos. Posteriormente, las normas que regularon el plan de beneficios (Acuerdo 03 de 2009, Acuerdo 08 de 2009, Acuerdo 028 de 2011, Acuerdo 029 de 2011, Resolución 5521 de 2013, Resolución 5592 de 2015, Resolución 6408 de 2016, Resolución 5269 de 2017 y Resolución 5857 de 2018), consagraron siempre un listado de exclusiones, e incluso, establecieron que las reintervenciones estarían cubiertas por el plan, siempre y cuando el procedimiento inicial no fuese alguno de los excluidos.

Estas exclusiones quedaron finalmente contempladas en el Artículo 15 de la Ley 1751 de 2015 (Estatutaria de Salud), donde se consagraron una serie de prestaciones que no podrían ser cubiertas con los recursos del sistema:

En todo caso, los recursos públicos asignados a la salud no podrán destinarse a financiar servicios y tecnologías en los que se advierta alguno de los siguientes criterios:

a) Que tengan como finalidad principal un propósito cosmético o suntuario no relacionado con la recuperación o mantenimiento de la capacidad funcional o vital de las personas;

b) Que no exista evidencia científica sobre su seguridad y eficacia clínica;

c) Que no exista evidencia científica sobre su efectividad clínica; 
d) Que su uso no haya sido autorizado por la autoridad competente;

e) Que se encuentren en fase de experimentación;

f) Que tengan que ser prestados en el exterior. (Ley 1751, 2015)

No obstante, pese a que la normatividad ha contemplado estrictamente las exclusiones del plan de beneficios, la jurisprudencia de la Corte Constitucional ha estudiado el tema, y ha establecido reglas para que se exceptúe el criterio de exclusión. Por tal motivo, se plantea la necesidad de revisar las líneas que se han desarrollado en torno al tópico de las exclusiones, para así visualizar su tendencia y conocer el entorno jurídico en el que los actores del sistema se ven involucrados. Para ello, se estudiarán los planteamientos de la Corte en torno a los literales a, d, e y f del citado Artículo 15, pues los literales b y c han sido discutidos al tratar el literal d sobre la prevalencia de la evidencia científica frente a la autorización por la autoridad competente.

Para la revisión de las líneas de decisión, se utilizó una metodología de ingeniería inversa, a través de la cual se toma una sentencia reciente de la Corte Constitucional colombiana, y a partir de ella se rastrean cuáles son las sentencias que esta cita para sustentar y consolidar su posición. De esa forma se puede encontrar una línea de decisiones por medio de la cual se verifica la postura que ha tenido la alta corporación frente a un tema puntual.

\section{Servicios y tecnologías con propósito cosmético o suntuario}

La Ley 1751 de 2015 estableció que no serían cubiertas, con cargo a los recursos del sistema de salud, aquellas prestaciones que "tengan como finalidad principal un propósito cosmético o suntuario no relacionado con la recuperación o mantenimiento de la capacidad funcional o vital de las personas". Así, salvo que exista un concepto médico que indique que una prestación en inicio cosmética, es necesaria para conservar o recuperar la funcionalidad o preservar la vida de alguien, esta no podría realizarse con cargo al sistema de salud.

No obstante, la Corte Constitucional ha tomado en cuenta otros elementos por fuera de la recuperación de funcionalidad o conservación de la vida para ordenar la autorización de procedimientos inicialmente estéticos, bajo el 
entendido de que también hay factores psíquicos, emocionales y sociales que hacen necesaria la prestación; así lo sostuvo en la sentencia T-159 de 2015:

(...) no siempre las intervenciones estéticas tienen fines cosméticos o de embellecimiento y, por consiguiente, no todos los procedimientos estéticos pueden tenerse en tanto excluidos del Plan Obligatorio de Salud. Aquellas intervenciones orientadas a restablecer la apariencia normal de las personas se ligan estrechamente con el reconocimiento de su dignidad y con la necesidad de no vulnerar tal dignidad, se consideran incluidas en el Plan Obligatorio de Salud y no pueden catalogarse como intervenciones superfluas con fines de embellecimiento. De modo que el derecho a la salud y a la vida digna no se limita únicamente al carácter funcional y físico, sino que abarca el aspecto psíquico, emocional y social de la persona. (Corte Constitucional, sentencia T-159, 2015).

De esta forma, la Corte ha ampliado las consideraciones por tener en cuenta al momento de analizar si una cirugía estética se encuentra excluida o no, yendo más allá de los aspectos funcionales y vitales. Esta postura se ha construido de manera firme en múltiples sentencias como la T-026 de 2011, T-1176 de 2008, T-307 de 2006, T-659 de 2003, T-461 de 2001, T-119 de 2000, T-572 de 1999 y la T-102 de 1998.

En las citadas providencias la Corte Constitucional ha estudiado casos de cirugías estéticas cuya autorización fue negada por las EPS, aduciendo que se trataba de procedimientos cosméticos o suntuarios, con los cuales no se buscaba la recuperación de la capacidad funcional de algún órgano ni evitar un riesgo para la vida, motivo por el cual se encontraban todos excluidos de la financiación a cargo del sistema de salud.

Frente a ello, la Corte asumió que no solo se podía considerar la necesidad de recuperar la capacidad funcional o evitar riesgos vitales, sino que se debía tener en perspectiva los aspectos psicológicos, morales y sociales que rodeaban cada caso, con lo cual se evidenció que los accionantes manifestaban afectaciones al desarrollo normal de su vida por la apariencia que presentaban, e incluso, afirmaban sentirse mal en su parte psíquica.

A continuación, se resumen los casos estudiados por la Corte, y se puntualiza el tipo de cirugía que se pidió, el diagnóstico que presentaba la persona y por el cual pedía la cirugía y, finalmente, la decisión: 
Tabla 1. Recuento de los casos estudiados

\begin{tabular}{|c|c|c|c|}
\hline SENTENCIA & CIRUGÍA & DIAGNÓSTICO & RESUELVE \\
\hline T-102/1998 & $\begin{array}{l}\text { Mamoplastia } \\
\text { bilateral }\end{array}$ & $\begin{array}{l}\text { Hipertrofia mamaria bilateral, cifosis- } \\
\text { lordosis en mayor de edad }\end{array}$ & Concede \\
\hline T-572/1999 & $\begin{array}{l}\text { Implante de } \\
\text { prótesis mamarias }\end{array}$ & $\begin{array}{l}\text { Enfermedad fibroquística de seno en } \\
\text { mayor de edad }\end{array}$ & Concede \\
\hline T-119/2000 & $\begin{array}{l}\text { Mamoplastia } \\
\text { reductora }\end{array}$ & Hiperplasia mamaria bilateral & Concede \\
\hline T-461/2001 & $\begin{array}{l}\text { Mamoplastia } \\
\text { reductora }\end{array}$ & $\begin{array}{l}\text { Hipertrofia mamaria en mayor de } \\
\text { edad }\end{array}$ & Concede \\
\hline$T-659 / 2003$ & $\begin{array}{l}\text { Ginecomastia } \\
\text { puberal colateral }\end{array}$ & $\begin{array}{l}\text { aumento del tamaño mamario } \\
\text { bilateral que le ocasiona apariencia } \\
\text { femenina a un menor de edad }\end{array}$ & Concede \\
\hline T-307/2006 & Otoplastia bilateral & $\begin{array}{l}\text { hiplasia del tercio superior de la oreja } \\
\text { en menor de edad }\end{array}$ & Concede \\
\hline$T-1176 / 2008$ & $\begin{array}{l}\text { Implante de } \\
\text { prótesis mamarias }\end{array}$ & $\begin{array}{l}\text { Extirpación de tumor maligno en } \\
\text { mayor de edad }\end{array}$ & Concede \\
\hline T-026/2011 & $\begin{array}{l}\text { Lipoinyección en } \\
\text { cara }\end{array}$ & $\begin{array}{l}\text { Alteración de contorno facial por } \\
\text { resección de tumor benigno de } \\
\text { maxilar inferior derecho, en mayor de } \\
\text { edad }\end{array}$ & Concede \\
\hline T-159/2015 & Blefaroplastia & $\begin{array}{l}\text { Trastorno del nervio facial, parálisis } \\
\text { facial periférica secundaria a } \\
\text { mastoidectomía y mastoiditis, en } \\
\text { mayor de edad. }\end{array}$ & $\begin{array}{l}\text { Ordena realizar una nueva } \\
\text { evaluación en el CTC, } \\
\text { argumentando el motivo en caso } \\
\text { de negativa }\end{array}$ \\
\hline T-159/2015 & $\begin{array}{l}\text { Abdominoplastia } \\
\text { circunferencial }\end{array}$ & $\begin{array}{l}\text { Obesidad mórbida y lipodistrofia } \\
\text { abdominal universal }\end{array}$ & $\begin{array}{l}\text { Ordena realizar una nueva } \\
\text { evaluación en el CTC, } \\
\text { argumentando el motivo en caso } \\
\text { de negativa }\end{array}$ \\
\hline
\end{tabular}

Fuente: Elaboración del autor.

De lo anterior se puede concluir que, pese a que la Ley Estatutaria de Salud excepcionó la exclusión de cirugías estéticas, solo para los casos donde se buscara la recuperación o mantenimiento de la capacidad funcional y vital de las personas, la Corte Constitucional ha ampliado esa excepción para los casos donde exista una afectación psíquica, moral o social.

Esto implica que, en aquellos casos donde se busque una cirugía para corregir situaciones físicas que generen una afectación psíquica o social para 
la persona, la Corte aplicaría una excepción a la exclusión y ordenaría cubrir el procedimiento. Sin embargo, el criterio de afectación "psíquica, moral y social" puede llegar a ser muy subjetivo, lo cual genera no solo inseguridad jurídica sobre los casos donde se podría aplicar la exclusión, sino también la posibilidad de que la cobertura de este tipo de procedimientos se vea ampliada drásticamente.

\subsection{Complicaciones de procedimientos cosméticos o suntuarios}

Dentro de las exclusiones por procedimientos estéticos con fines cosméticos o suntuarios, existe también una restricción frente a las reintervenciones producto de las complicaciones de estos. Al respecto, desde el Acuerdo 08 de 2009 ya se había establecido que el plan de beneficios cubría las reintervenciones, siempre y cuando el procedimiento inicial no hubiese sido parte de las exclusiones, disposición que se mantuvo - aunque con una redacción distinta - en las normas posteriores del plan de beneficios.

La sentencia T-579 resolvió el caso de dos mujeres a las cuales se les negó el cubrimiento de servicios, aduciendo que eran complicaciones de cirugías estéticas y, por lo tanto, les aplicaba la exclusión, por lo cual los gastos de tales complicaciones tuvieron que asumirse de manera privada.

Para la Corte, cabe realizar una distinción entre la cirugía meramente cosmética y la cirugía funcional. Basándose en esta diferencia, llega a concluir que:

(...) los efectos secundarios o complicaciones derivadas de una cirugía estética comprometen muy gravemente la funcionalidad de los órganos o tejidos del cuerpo que no fueron objeto de dicha cirugía inicial, esa circunstancia desborda el alcance de lo que podría entenderse como efectos secundarios o complicaciones previstas científicamente para cada tipo de cirugía estética (...). (Corte Constitucional, sentencia T-579, 2017)

Y continúa:

El supuesto que se acaba de plantear corresponde al caso en que se encuentra severamente comprometida la funcionalidad de la parte del cuerpo que originalmente fue intervenida con fines netamente estéticos, pero cuyos complicaciones impactan gravemente su funcionalidad y la de otros órganos que no fueron objeto del tratamiento estético inicial, y 
que de no ser atendidos medicamente (SIC) de manera oportuna y eficaz, podría llevar al compromiso serio de la salud o de la vida misma. (...) (Corte Constitucional, sentencia T-579, 2017).

Bajo esa perspectiva, para la Corte es posible asumir tratamientos que se deriven de complicaciones de cirugías estéticas, siempre y cuando no estuviesen dentro de los riesgos previstos o inherentes a las mismas, en cuyo caso quedan bajo el ámbito de exclusión y deberán ser asumidos por el paciente.

Esta sentencia plantea un cambio en la postura que había venido manifestando la Corte Constitucional en sentencias anteriores, toda vez que reconoce la posibilidad de que complicaciones de cirugía estéticas sean cubiertas por el Plan de Beneficios, cuando en otros pronunciamientos no lo había permitido. Valga aclarar que la Corte sí había concedido la realización de cirugías, en principio estéticas, cuando encontraba que en realidad tenían componentes funcionales. Sin embargo, en el caso de complicaciones de cirugías meramente estéticas no había otorgado el amparo.

Un ejemplo de lo anterior se encuentra en la sentencia T-676, en la cual se estudió el caso de una mujer que ingresó a una clínica con el fin de practicarse una cirugía plástica de aumento de senos, lipectomía abdominal y lipoinyección glútea, pero luego de realizada la operación se presentaron una serie de complicaciones como trombosis arterial, isquemía severa e insuficiencia renal, lo cual puso en peligro la integridad de sus miembros inferiores, uno de los cuales, al momento de la presentación de la acción, ya había sido amputado desde la cadera, y en el otro se le amputaron parcialmente los dedos; además, tuvo complicaciones en su sistema respiratorio y riñones. Frente a esta situación, el médico ordenó la práctica de un tratamiento de hemodiálisis y recuperación vascular, procedimientos denegados por ser complicaciones de una cirugía estética.

En tal ocasión, la Corte manifestó que el estado de salud se encontraba afectado por las consecuencias de una cirugía estética a la cual la persona se sometió libre y voluntariamente. Incluso, llegó a considerar:

En derecho, nadie puede invocar su propia culpa para beneficiarse y menos aún para pretender que el Estado asuma los gastos por concepto de atención médica requerida por la situación de riesgo en que la misma persona se ha colocado por imprudencia, pues debió prever las consecuencias que traería 
el sometimiento a este tipo de cirugías, cuyas complicaciones y desenlaces fatales para la vida son, hoy por hoy, de público conocimiento. De asumir el estado (SIC) dichos costos, ello implicaría a su vez una disminución de los recursos destinados a atender la salud de la población pobre y vulnerable. (Corte Constitucional, sentencia T-676, 2002).

De igual forma, se pronunció en la sentencia T-793, donde estudió la solicitud de cambio de prótesis mamaria que había solicitado una mujer, pues tenía diagnóstico de ruptura y recomendación del médico de realizar el procedimiento para evitar una afectación mayor. La Corte determinó que la ruptura era uno de los riesgos inherentes de la cirugía estética, y afirmó que “(...) la demandante desde el momento en el cual decidió de manera voluntaria y con un criterio estético, someterse a este procedimiento, sin previa autorización por parte de la EPS., asumía las consecuencias emanadas de su decisión”, por lo cual negó el amparo solicitado (2010).

\section{Uso no autorizado por la autoridad competente: medicamentos sin indicación o registro del Invima}

Inicialmente, se expidió la Resolución 5061 de 1997, por medio de la cual se reglamentaron los comités técnico-científicos (CTC), y en el literal d) de su artículo $4 .^{\circ}$ se estipuló que solo podrían prescribirse medicamentos debidamente autorizados para su comercialización y expendio en el país. Posteriormente, el uso de fármacos que no estuviesen autorizados por la autoridad competente (Invima), fue establecido como una de las exclusiones del plan de beneficios, en el literal b) del artículo $6^{\circ}$ del Acuerdo 028 de 2011 de la Comisión de Regulación en Salud (Cres). Similar disposición fue incorporada en las demás normas que actualizaron el plan de beneficios, hasta que fue incluso consagrada la exclusión en la Ley Estatutaria de Salud.

Lo anterior derivó en que solo se autorizaran aquellos medicamentos que contaban con registro vigente ante el Invima y cuya indicación coincidiera con la patología por tratar; esto llevó a que algunos pacientes recurrieran a la acción de tutela como mecanismo para que se le ordenara autorizar y suministrar los medicamentos prescritos por el médico tratante. Fue entonces cuando la Corte Constitucional asumió el tema al revisar algunos 
casos de medicamentos sin indicación o registro Invima, y comenzó a resolver en abundante jurisprudencia excepcionando esta exclusión del sistema.

Ahora bien, es preciso aclarar la diferencia existente entre los conceptos registro e indicación, pues los mismos pueden verse erróneamente utilizados en diversos espacios y aun la misma Corte los suele usar de manera indistinta. Para lo anterior, se deben tener en cuenta las definiciones establecidas en el artículo 2. ${ }^{\circ}$ del Decreto 677 de 1995 :

(...) Indicaciones: estados patológicos o padecimientos a los cuales se aplica un medicamento.

Registro sanitario: es el documento público expedido por el Invima o la autoridad delegada, previo el procedimiento tendiente a verificar el cumplimiento de los requisitos técnicolegales establecidos en el presente decreto, el cual faculta a una persona natural o jurídica para producir, comercializar, importar, exportar, envasar, procesar y/o expender los medicamentos cosméticos, preparaciones farmacéuticas a base de recursos naturales, productos de aseo, higiene y limpieza y otros productos de uso doméstico.

Hasta ese punto, resultaba claro que, a partir de las disposiciones reglamentarias, no era posible autorizar y suministrar medicamentos sin indicación o registro Invima. Frente a esta situación, la jurisprudencia constitucional determinó detener la aplicación de la normatividad y ordenar la autorización y suministro de los medicamentos, siempre y cuando se cumpliera, en el caso concreto, con una serie de requisitos.

Al respecto, la sentencia T-302 expresó que se ha sentado una regla jurisprudencial frente a la orden por vía de tutela de medicamentos sin registro sanitario Invima, donde serán procedentes siempre y cuando sean medicamentos acreditados por la comunidad científica frente a su idoneidad para el tratamiento de una patología, y además cumpla con los requisitos para ordenar elementos no contemplados en el plan de beneficios, a saber: que la falta del medicamento ponga en riesgo los derechos fundamentales a la vida o la salud del paciente; que no puedan ser sustituidos por otros contemplados en el plan de beneficios; que no puedan ser adquiridos directamente por el paciente; y que hayan sido prescritos por el médico tratante, sin que necesariamente este deba estar adscrito a la EPS (2014). 
De esta forma, un rastreo jurisprudencial en las providencias de la Corte Constitucional permite identificar un total de 18 sentencias, en las cuales se manifiesta claramente una postura uniforme en cuanto a la autorización de suministro de medicamentos que no cuenten con el aval de la autoridad nacional para su uso en determinada patología, siempre y cuando se sigan las reglas ya mencionadas.

Con este rastreo se encontró una línea compuesta por las sentencias T-975 de 1999, T-344 de 2002, T-173 de 2003, T-884 de 2004, T-945 de 2004, T-1328 de 2005, T-297 de 2005, T-1214 de 2008, T-706 de 2010, T-418 de 2011, T-834 de 2011, T-042 de 2013, T-425 de 2013, T-061 de 2014, T-302 de 2014, C-313 de 2014, T-105 de 2015 y T-243 de 2015.

También se encontraron dos pronunciamientos más recientes, los cuales confirmaron la línea de decisión, pero no hicieron parte de la misma en su ingeniería inversa por no tener mayor impacto: T-313 de 2015 y T-001 de 2018.Con la revisión efectuada, se procedió a estructurar el siguiente gráfico, para así dimensionar el esquema que ha desarrollado la Corte con sus providencias:

\section{Gráfico 1. Nicho citacional}

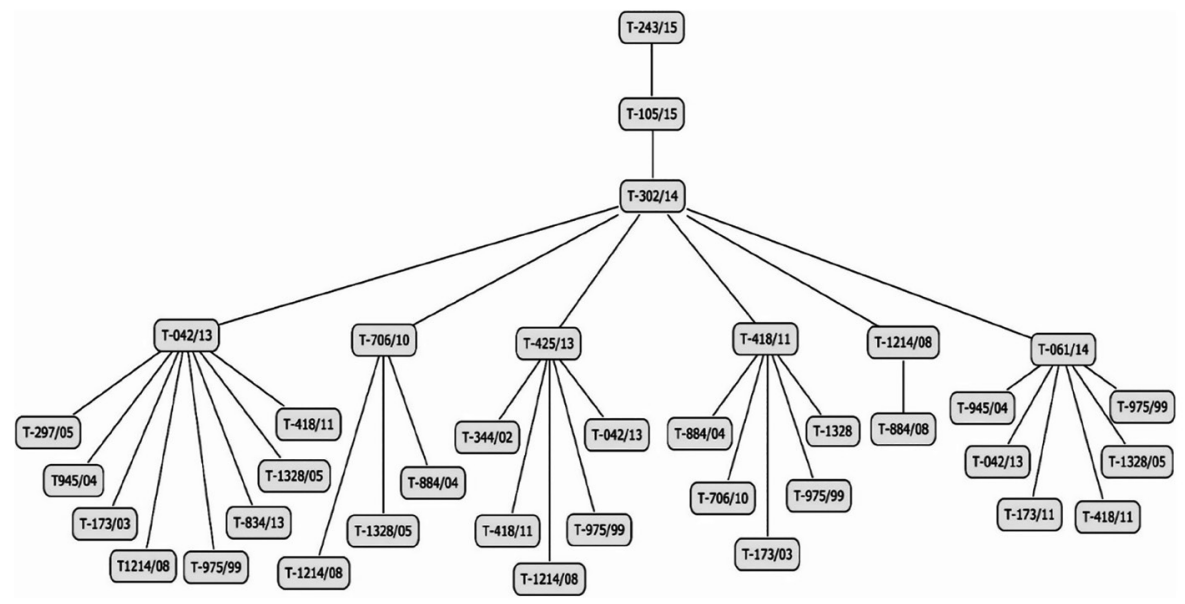

Fuente: Elaboración del autor. 
Por otro lado, tras revisar cada una de las sentencias de tutela, se encontró que fueron autorizados los siguientes medicamentos para determinadas patologías:

Tabla 2. Medicamentos ordenados

\begin{tabular}{|c|c|c|c|}
\hline SENTENCIA & MEDICAMENTO & DIAGNÓSTICO & RESUELVE \\
\hline T-975/1999 & $\begin{array}{l}\text { Dimetilsulfóxido } \\
\text { solución (Rimso) }\end{array}$ & Cistitis intersticial & Concede \\
\hline$T-344 / 2002$ & Etanerceot & Artritis reumatoide & Concede \\
\hline$T-173 / 2003$ & Elmiron & Cistitis crónica activa & Concede \\
\hline$T-884 / 2004$ & Sinaxial de $100 \mathrm{mg}$ & $\begin{array}{l}\text { Trauma en columna cervical en } \\
\text { extensión, presentando paraplejía } \\
\text { inmediata y diparesia braquial distal }\end{array}$ & Concede \\
\hline$T-945 / 2004$ & Dimetil sulfóxido & Cistitis crónica & $\begin{array}{l}\text { Se abstiene de ordenarlo, pues } \\
\text { considera que su uso no está } \\
\text { avalado para la patología. }\end{array}$ \\
\hline T-1328/2005 & Thyrogen & Cáncer de garganta & Concede \\
\hline T-297/2005 & $\begin{array}{l}\text { Primidona } \\
\text { (mysoline) }\end{array}$ & Convulsiones & $\begin{array}{l}\text { No concede, pues aunque no está } \\
\text { disponible el medicamento, } \\
\text { existen sustitutos. }\end{array}$ \\
\hline T-1214/2008 & Rituximab & Pseudotumor orbitario & Concede \\
\hline T-706/2010 & $\begin{array}{l}\text { Inmunoterapia } \\
\text { bacteriana bacterius } \\
\text { AB en gotas }\end{array}$ & $\begin{array}{l}\text { Infecciones respiratorias recurrentes en } \\
\text { menor intervenida de adenoides }\end{array}$ & Concede \\
\hline T-418/2011 & Bevacizumab (Avastin) & $\begin{array}{l}\text { Retinopatía diabética proliferativa } \mathrm{AO} \\
\text { con edema macular }\end{array}$ & Concede \\
\hline T-834/2011 & $\begin{array}{l}\text { Inmunoterapia } \\
\text { subcutánea }\end{array}$ & Alergia a múltiples sustancias & Concede \\
\hline $\mathrm{T}-042 / 2013$ & $\begin{array}{l}\text { Micofenolato de } \\
\text { mofetil }\end{array}$ & $\begin{array}{l}\text { Lupus eritematoso sistémico con } \\
\text { compromiso renal }\end{array}$ & Concede \\
\hline$T-425 / 2013$ & Hidroxiurea & Anemia falciforme & Concede \\
\hline$T-061 / 2014$ & $\begin{array}{l}\text { Micofenolato de } \\
\text { mofetil }\end{array}$ & $\begin{array}{l}\text { Lupus eritematoso sistémico con } \\
\text { compromiso de órganos y sistemas }\end{array}$ & Concede \\
\hline$T-302 / 2014$ & $\begin{array}{l}\text { Cellcepto Micofenolato } \\
\text { de mofetil e Hydroclor }\end{array}$ & Esclerodermia sistémica difusa & Concede \\
\hline$T-105 / 2015$ & $\begin{array}{l}\text { Micofenolato de } \\
\text { mofetil }\end{array}$ & $\begin{array}{l}\text { Atopia severa con marcado compromiso } \\
\text { de piel, de origen inmunológico con } \\
\text { eosinofilia progresiva, sin respuesta a } \\
\text { tratamiento con prednisolona }\end{array}$ & Concede \\
\hline$T-243 / 2015$ & $\begin{array}{l}\text { Inhibidor } \mathrm{CL} \text { esterasa } \\
\text { (cinryze) }\end{array}$ & Edema angioneurótco & Concede \\
\hline
\end{tabular}

Del listado llama la atención la contradicción entre las sentencias T-975 de 1994 y la T-945 de 2004, en las cuales se revisaron las solicitudes del medicamento Dimetil sulfóxido, determinando concederlo en la primera y 
negarlo en la segunda por falta de soporte científico, pese a que se estaba frente a la misma patología de cistitis.

Teniendo en cuenta este panorama jurisprudencial, el Ministerio de Salud y Protección Social impulsó un cambio importante en el sistema al expedir la Resolución 3951 de 2016 (sustituida por la Resolución 1885 de 2018), a través de la cual se eliminó el CTC en el régimen contributivo, se configuró un sistema para la prescripción de medicamentos no cubiertos por el plan de beneficios y, se permitió la autorización de medicamentos sin indicación del Invima bajo ciertos requisitos.

De acuerdo con la nueva reglamentación, cuando el médico considere que se debe ordenar un medicamento que no cuente con indicación o registro del Invima para la patología de su paciente, pero según la evidencia científica sea la mejor opción, podrá realizar la prescripción a través de la plataforma virtual (MIPRES), siempre y cuando su uso esté reportado por las sociedades científicas ante el Ministerio de Salud y se encuentre incluido en el listado de usos no incluidos en registro sanitario (UNIRS). Una vez ordenado el medicamento, la solicitud será revisada por una junta de profesionales de la salud, donde se definirá si finalmente se autoriza o no la prescripción.

\section{Servicios y tecnologías en fase de experimentación: derecho a que sea intentado}

Otra de las exclusiones que se encuentran en la Ley Estatutaria de Salud es la relacionada con el financiamiento de servicios y tecnologías que estén en fase de experimentación, por lo que se estableció que no deben ser cubiertos por los recursos del sistema de salud. Esta exclusión fue determinada, incluso, desde la Resolución 5261 de 1994, y ubica entre los limitantes del plan de beneficios a los tratamientos de carácter experimental.

No obstante, pese a que la normatividad ha sido clara en cuanto a la exclusión de tratamientos experimentales, la Corte Constitucional de Colombia, en la sentencia T-057, introdujo, por primera vez en Colombia, una figura del derecho estadounidense: el denominado right to try (2015). 
La citada sentencia ha sido el único precedente en la Corte Constitucional, y en ella se introdujo la figura del right to try o derecho a ensayar, a experimentar, al analizar el caso de una joven de 29 años que se encontraba en estado vegetativo persistente (vigil), a quien su médico tratante le ordenó una estimulación espinal epidural cervical, como única alternativa de tratamiento para mejorar su calidad de vida. Sin embargo, el tratamiento no fue autorizado por la EPS, toda vez que tenía un carácter experimental, conclusión que fue reiterada por especialistas de otra IPS, quienes afirmaron que no era una opción de manejo por los malos resultados obtenidos en estudios clínicos, y además los equipos usados para ello, no contaban con aprobación de la FDA (Food and Drug Administration) y mucho menos del Invima.

Bajo ese panorama, la Corte consideró posible incorporar, por vía del Artículo 94 de la Constitución Política de 1991, el derecho fundamental a que sea intentado, basándose en la figura del right to try que se ha desarrollado en Estados Unidos para los casos de pacientes con enfermedades terminales, para quienes no existe una alternativa de tratamiento diferente a uno de carácter experimental. Resalta la sentencia que, para la aplicación de esta figura en Estados Unidos, se deben cumplir ciertas condiciones:

(I) (a) "paciente elegible" significa una persona que tiene: una enfermedad terminal, atestiguada por médico tratante del paciente;

(ii) tomando en cuenta otras opciones de tratamiento actualmente aprobadas por la FDA;

(iii) haber recibido una recomendación de su médico para un fármaco en investigación, productos biológicos o dispositivo;

(iv) (SIC) haber suministrado por escrito, el consentimiento informado para el uso de la droga en investigación, productos biológicos o dispositivo o, si el paciente es menor de edad o carece de la capacidad mental para dar su consentimiento informado, un padre o tutor legal haya dado el consentimiento informado por escrito en nombre del paciente.

Y en el caso concreto de la citada sentencia, la Corte concluye:

(...) la Sala encuentra que: (i) se está ante la única y quizá última opción para una paciente, dado que, salvo el Dr. Páez Novoa, ningún médico ha propuesto o planteado alternativa alguna de recuperación para la joven Mairoby; (ii) se cuenta con ciertos fundamentos científicos para afirmar que no se está ante un tratamiento completamente desconocido o novedoso, así las autoridades correspondientes, nacionales o extranjeras, todavía no 
lo hayan aprobado; (iii) los riesgos del tratamiento han sido explicados a los familiares, los cuales los han asumido; y (iv) el médico que le ha venido haciendo un seguimiento permanente a la evolución de la paciente, tiene la firme convicción de que el tratamiento propuesto brinda una "luz de esperanza” en la materia.(Corte Constitucional, T-057, 2015).

De lo planteado, se puede apreciar que el derecho a que sea intentado, es básicamente un nuevo derecho fundamental descrito por la Corte, para permitir que el sistema de salud autorice y cubra tratamientos experimentales en aquellos casos donde un paciente terminal o en estado vegetativo persistente no cuente con otras posibilidades de tratamiento, según su médico tratante, y se haya aceptado voluntaria y conscientemente los riesgos que conlleva utilizar esto como última alternativa.

\section{Servicios que deban ser prestados en el exterior}

La última exclusión del plan de beneficios que consagró la Ley Estatutaria de Salud consiste en que los servicios tengan lugar en el exterior; esto, atendiendo al costo que puede generar asumir prestaciones en otros países, y a un criterio de territorialidad para la cobertura del sistema, pues desde la formulación en la Ley 100 de 1993, ha sido claro que el sistema cubre a los residentes del país dentro de los límites geopolíticos del mismo.

No obstante, para esta exclusión, la Corte Constitucional también ha manifestado que es posible flexibilizarla, y de hecho, al declarar la constitucionalidad de su consagración en la Ley Estatutaria de Salud a través de la sentencia C-313, estableció que "se trata de un criterio, sujeto a ser inaplicado en los casos y con las condiciones que la jurisprudencia constitucional ha indicado" (2014).

En la sentencia T-279 de 2017, su pronunciamiento más reciente, reconstruye la línea de decisión que ha formado, constituida por las sentencias T-165 de 1995, T-645 de 1996, T-304 de 1998, T-395 de 1998, SU-819 de 1999, T-597 de 2001, T-613 de 2012 y T-180 de 2013. Indica, igualmente en tal providencia, que a lo largo de dicha línea se han construido criterios para excepcionar la exclusión de tratamientos en el exterior, los cuales pueden sintetizarse en: 
En conclusión, el Sistema de Seguridad Social en Salud, por regla general, no garantiza la prestación de servicios médicos que se lleven a cabo en el extranjero. Sin perjuicio de ello, la jurisprudencia ha indicado que la exclusión en mención no es admisible cuando está en riesgo la vida del afiliado. En estos casos, se debe demostrar que el procedimiento no puede ser realizado en Colombia, que el tratamiento no es de carácter experimental, que no se tiene la capacidad de pago para costear el mismo y, adicionalmente, que se cuenta con concepto favorable del médico tratante, en el que se indique la eficacia y el beneficio para la salud derivado del procedimiento, esto es, por qué se requiere con urgencia. Por supuesto, sin descartar otros aspectos particulares y específicos que un caso de esta naturaleza pueda presentar. (Corte Constitucional, T-279, 2017)

En ese orden de ideas, la Corte ha establecido, para este criterio de exclusión, una clara línea de decisión acerca de los casos donde se debe excepcionar. Así, el principio de territorialidad del sistema de salud termina cediendo frente a tratamientos en el exterior si son vitales, no experimentales y no tienen un sustituto en el país. Sin embargo, como también lo manifestó la Corte en la sentencia la SU-819 de 1999, la más importante de esta línea, por ser una providencia de unificación con los criterios y análisis más completos: "Todos los procedimientos o exámenes que se puedan realizar en Colombia deben respetar el principio de la territorialidad del sistema”.

\section{Conclusiones}

- Pese a que en la Ley Estatutaria de Salud ya se hubiese establecido la diferencia entre un fin suntuario y uno funcional en las cirugías estéticas, la corte ha ido más allá en sus providencias, estableciendo que no solo debe tenerse en cuenta la rehabilitación funcional para cubrir los procedimientos estéticos, sino que es necesario verificar que no exista una afectación psíquica, moral o social, pues de ser así no podría aplicarse la exclusión, en aras de brindar una atención integral a las personas.

- Ante la inclusión jurisprudencial de la afectación psíquica, moral o social, cabe preguntarse si normalmente detrás del deseo de realizarse una intervención con fin suntuario, existe una inconformidad subjetiva de la persona frente a alguna parte de su cuerpo, que le genere una afectación psíquica o social. De esta forma, se podría estar abriendo una brecha 
muy grande para que el sistema de salud, con recursos limitados, termine cubriendo cirugías para corregir la apariencia de las personas.

- En el pronunciamiento más reciente de la Corte sobre reintervenciones de cirugías estéticas con finalidad suntuaria, se cambió la postura que había venido sosteniendo, al determinar que, en algunos casos, sí se deben cubrir las reintervenciones por parte del sistema de salud. Esto, para los casos donde la reintervención ocurre por complicaciones que no se podían esperar o no eran inherentes al procedimiento, y además, hay compromiso de órganos que no hacían parte de la operación estética.

- La jurisprudencia constitucional ha venido inaplicando las normas que impiden la autorización de prestaciones que no contaran con el registro o la indicación para determinadas patologías por parte del Invima. Así, ante el conflicto entre los trámites administrativos de registro con el criterio científico de los médicos tratantes, la Corte determinó que primaba el segundo.

- Ante la clara tendencia de la Corte Constitucional sobre la forma de excepcionar esta exclusión, manifestada en una abundante producción de sentencias, y la presión que implica el choque entre el criterio médicocientífico y el trámite administrativo, el Ministerio de Salud determinó, por medio de la Resolución 3951 de 2016, establecer un procedimiento para que este tipo de prestaciones fuesen autorizadas, e incluso estableció la creación de un listado de usos no incluidos en registro sanitario (UNIRS), para facilitar su autorización.

- En una decisión que no ha tenido reiteraciones, la Corte Constitucional introdujo en Colombia la figura del right to try estadounidense, por medio del Artículo 94 sobre derechos innominados de la Constitución Política de 1991, determinando así que el "derecho a que sea intentado" haga parte de los derechos fundamentales por su relación con la dignidad humana.

- De acuerdo con la Corte, se aplicaría el derecho a intentar, a experimentar, en el caso de pacientes con enfermedades terminales o en estado vegetativo persistente, cuando un tratamiento experimental sea la única alternativa, cuando exista suficiente convencimiento del médico tratante acerca de su viabilidad y se cuente con el consentimiento informado del paciente o de su representante legal. 
- Ante la tensión entre la territorialidad y el derecho a la salud, la Corte determinó que debe primar el segundo; sin embargo, siempre prevalecerá el primero en los casos donde el servicio pueda prestarse en Colombia.

A continuación, se presentan gráficamente las tendencias encontradas en la jurisprudencia de la Corte Constitucional:

Gráfica 2. Tendencias de la jurisprudencia constitucional sobre exclusiones del plan de beneficios.

\section{TENDENCIAS DE LA JURISPRUDENCIA CONSTITUCIONAL} SOBRE EXCLUSIONES DEL PLAN DE BENEFICIOS
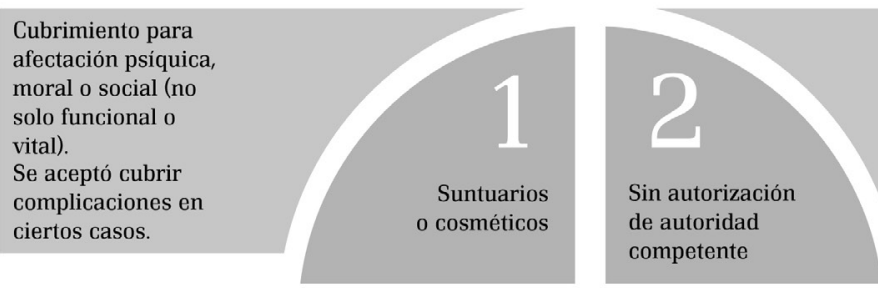

Prevalencia de la evidencia científica sobre el registro del Invima.

Se positivizó la jurisprudencia y se creó listado UNIRS.

Se incorporó el right to try: derecho fundamental a intentar tratamientos experimentales

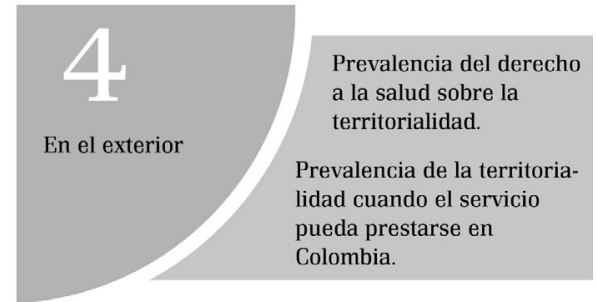

Fuente: Elaboración del autor.

\section{Referencias}

Congreso de la República. (1993) Por la cual se crea el sistema de seguridad social integral y se dictan otras disposiciones, Ley 100. Recuperado de https://www. minsalud.gov.co/Normatividad_Nuevo/LEY\%200100\%20DE\%201993.pdf

Congreso de la República. (2015). Por medio de la cual se regula el derecho fundamentala la salud y se dictan otras disposiciones, Ley 1751. Recuperado de https://www. minsalud.gov.co/Normatividad_Nuevo/Ley\%201751\%20de\%202015.pdf

Corte Constitucional. (1995). Bogotá. Sentencia T-165. Magistrado ponente: Vladimiro Naranjo Mesa. 
Corte Constitucional. (1996). Bogotá. Sentencia T-645. Magistrado ponente Alejandro Martínez Caballero.

Corte Constitucional. (1998).Bogotá. Sentencia T-102. Magistrado ponente: Antonio Barrera Carbonell.

Corte Constitucional. (1998). Bogotá. Sentencia T-304. Magistrado ponente: Fabio Morón Díaz.

Corte Constitucional. (1998). Bogotá. Sentencia T-395. Magistrado ponente: Alejandro Martínez Caballero.

Corte Constitucional. (1999). Bogotá. Sentencia T-572. Magistrado ponente: Fabio Morón Díaz.

Corte Constitucional. (1999). Bogotá. Sentencia SU-819. Magistrado ponente:Álvaro Tafur Galvis.

Corte Constitucional. (1999). Bogotá. Sentencia T-975. Magistrado ponente: Álvaro Tafur Galvis.

Corte Constitucional. (2000). Bogotá. Sentencia T-119. Magistrado ponente: José Gregorio Hernández Galindo.

Corte Constitucional. (2000). Bogotá. Sentencia T-344. Magistrado ponente: José Gregorio Hernández Galindo.

Corte Constitucional. (2001). Bogotá. Sentencia T-461. Magistrado ponente: Marco Gerardo Monroy Cabra.

Corte Constitucional. (2001). Bogotá. Sentencia T-597. Magistrado ponente: Rodrigo Escobar Gil.

Corte Constitucional. (2002). Bogotá. Sentencia T-676 de 2002. Magistrado ponente: Jaime Araújo Rentería.

Corte Constitucional. (2003). Bogotá. Sentencia T-173. Magistrado ponente: Álvaro Tafur Galvis.

Corte Constitucional. (2003). Bogotá. Sentencia T-659. Magistrado ponente: Alfredo Beltrán Sierra.

Corte Constitucional. (2004). Bogotá. Sentencia T-884. Magistrado ponente: Humberto Sierra Porto. 
Corte Constitucional. (2004). Bogotá. Sentencia T-945. Magistrado ponente: Rodrigo Escobar Gil.

Corte Constitucional. (2005). Bogotá. Sentencia T-297. Magistrado ponente: Manuel José Cepeda Espinosa.

Corte Constitucional. (2005). Bogotá. Sentencia T-1328. Magistrado ponente: Humberto Antonio Sierra Porto.

Corte Constitucional. (2006). Bogotá. Sentencia T-307. Magistrado ponente: Humberto Antonio Sierra Porto.

Corte Constitucional. (2008). Bogotá. Sentencia T-1176. Magistrado ponente: Humberto Antonio Sierra Porto.

Corte Constitucional. (2008). Bogotá. Sentencia T-1214. Magistrado ponente: Humberto Antonio Sierra Porto.

Corte Constitucional. (2010). Bogotá. Sentencia T-706. Magistrado ponente: Gabriel Eduardo Mendoza Martelo.

Corte Constitucional. (2010). Bogotá. Sentencia T-793. Magistrado ponente: Jorge Iván Palacio Palacio.

Corte Constitucional. (2011). Bogotá. Sentencia T-206. Magistrada ponente: María Victoria Calle Correa.

Corte Constitucional. (2011). Bogotá. Sentencia T-418. Magistrada ponente: María Victoria Calle Correa.

Corte Constitucional. (2011). Bogotá. Sentencia T-834. Magistrada ponente: María Victoria Calle Correa.

Corte Constitucional. (2012). Bogotá. Sentencia T-613. Magistrado ponente: Jorge Ignacio Pretelt Chaljub.

Corte Constitucional. (2013). Bogotá. Sentencia T-042. Magistrado ponente Mauricio González Cuervo.

Corte Constitucional. (2013). Bogotá. Sentencia T-180. Magistrado ponente: Jorge Ignacio Pretelt Chaljub.

Corte Constitucional. (2013). Bogotá. Sentencia T-425. Magistrada ponente: María Victoria Calle Correa.

Corte Constitucional. (2014). Bogotá. Sentencia T-061. Magistrado ponente: Nilson Pinilla Pinilla. 
Corte Constitucional. (2014). Bogotá. Sentencia T-302. Magistrado ponente: Luis Guillermo Guerrero Pérez.

Corte Constitucional. (2014). Bogotá. Sentencia C-313. Magistrado ponente: Gabriel Eduardo Mendoza Martelo.

Corte Constitucional. (2015). Bogotá. Sentencia T-057. Magistrada ponente: Martha Victoria Sáchica Méndez.

Corte Constitucional. (2015). Bogotá. Sentencia T-105. Magistrada ponente: Gloria Stella Ortiz Delgado.

Corte Constitucional. (2015). Bogotá. Sentencia T-159. Magistrado ponente: Mauricio González Cuervo.

Corte Constitucional. (2015). Bogotá. Sentencia T-313. Magistrado ponente: Jorge Iván Palacio Palacio.

Corte Constitucional. (2017). Bogotá. Sentencia T-579. Magistrada ponente: Cristina Pardo Schlesinger.

Corte Constitucional. (2017). Bogotá. Sentencia T-279. Magistrado ponente: Aquiles Arrieta Gómez.

Corte Constitucional. (2018). Bogotá. Sentencia 001. Magistrada ponente: Cristina Pardo Schlesinger.

Resolución 5261 (1994). Por la cual se establece el manual de actividades, intervenciones y procedimientos del plan obligatorio de salud en el sistema general de seguridad social en salud. Ministerio de Salud. Recuperado de https://www.minsalud.gov.co/ Normatividad_Nuevo/RESOLUCI\%C3\%93N\%205261\%20DE\%201994.pdf

Resolución 5061 (1997) Por la cual se reglamentan los comités técnico- científicos dentro de la entidades promotoras de salud, administradoras del régimen subsidiado e instituciones prestadoras de servicios de salud, y se dictan otras disposiciones. Ministerio de Salud. Recuperado de https://www.minsalud.gov.co/Normatividad_ Nuevo/RESOLUCI\%C3\%93N\%205061\%20DE\%201997.pdf

Resolución 3951 (2016). Por la cual se establece el procedimiento de acceso, reporte de prescripción, suministro, verificación, control, pago y análisis de la información de servicios y tecnologías en salud no cubiertas por el Plan de Beneficios en Salud con cargo a la UPC y se dictan otras disposiciones, Ministerio de Salud y Protección Social. Recuperado de https://www.minsalud.gov.co/Normatividad_Nuevo/ Resoluci\%C3\%B3n\%203951\%20de\%202016.pdf 
Resolución 1885 (2018). Por la cual se establece el procedimiento de acceso, reporte de prescripción, suministro, verificación, control, pago y análisis de la información de servicios y tecnologías en salud no cubiertas por el Plan de Beneficios en Salud con cargo a la UPC y se dictan otras disposiciones, Ministerio de Salud y Protección Social. Recuperado de https://www.minsalud.gov.co/Normatividad_Nuevo/ Resolucion\%201885\%20de\%202018.pdf

Resolución 5857 (2018). Por la cual se actualiza integralmente el Plan de Beneficios en Salud con cargo a la Unidad de Pago por Capitación (UPC). Ministerio de Salud y Protección Social. Recuperado de https://www.minsalud.gov.co/Normatividad_ Nuevo/Resoluci\%C3\%B3n\%205857\%20de\%202018.pdf

Decreto 667 (1995). Por el cual se reglamenta parcialmente el régimen de registros y licencias, el control de calidad, así como el régimen de vigilancia sanitaria de medicamentos, cosméticos, preparaciones farmacéuticas a base de recursos naturales, productos de aseo, higiene y limpieza y otros productos de uso doméstico y se dictan otras disposiciones sobre la materia. Presidencia de la República. Recuperado de https://www.invima.gov.co/decretos-en-productosfitoterapeuticos/decreto-677-1995-pdf/download.html 